\title{
Intuitive Concept or Physical Meaning of Lagrangian
}

\author{
Susumu Morita \\ Department of Biofunctional and Mechanical Systems, Institute of Industrial Science, The University of Tokyo, \\ Tokyo, Japan \\ Email:morit@iis.u-tokyo.ac.jp
}

Received 24 February 2016; accepted 26 March 2016; published 29 March 2016

Copyright @ 2016 by author and Scientific Research Publishing Inc.

This work is licensed under the Creative Commons Attribution International License (CC BY). http://creativecommons.org/licenses/by/4.0/

(c) (i) Open Access

\section{Abstract}

The authors have been studying on the principle of motion generation behind animals, mainly human, and have reached a certain milestone with it in [1]. Because [1] ended up being very interdisciplinary, the author has been looking for an opportunity to close in on the part where we have grasped the conceptual idea of a Lagrangian. This paper proposes the physical meaning or its intuitive concept of a Lagrangian. This is a daring attempt because the topic is over 240 years of enigma, whereby so many have neglected of its absence, and physics has gone further towards its frontiers of their time, and has successfully flourished. Meanwhile, Lagrangian is not getting enough of teachers' attention on students getting stuck on this function, despite the fact that it is a strong foundation as is only the beginning towards Hamiltonian formalism, general relativity, and modern physics of today. This paper's sole motive is to answer what the title says in detail, helping each and everyone who faces Lagrangian for their first time. The paper is positioned to be a supplement for [1]. This literature had three topics bound into one. Out of the three, this document focuses in the part of the intuitive meaning of Lagrangian, since the paper had contents related to multiple disciplines. The author finds it worthy to discuss this topic in an independent, more detailed manner.

\section{Keywords}

Hamilton's Principle, Analytical Mechanics, Classical Mechanics, Lagrangian, Hamiltonian

\section{Introduction}

Lagrangian was introduced by Joseph Lagrange in his book Mèchanique Analytique in 1788. Back from then, this function has been told not to have a physical meaning, or something not to be asked for such, or neglect to 
even bother about its intuitive concept as being the majority attitude of distinctive textbooks. Vast amount of literature and reputable textbooks such as [2]-[5] have followed this stance, nonetheless played an important role of education given the long nearly 240 years of time spreading, innovating, and then flourished the technologies of today. Among many books, the author could only find [6] which states about the absence of a physical meaning to Lagrangian and the mysteriousness of it. Also, there are the more-than-countable literatures about Lagrangian, many on the application of Lagrangian to fields other than analytical mechanics. There are numbers of work focusing on the Lagrangian itself, but rather few compared to its applications. There is one of which is in the former group [7] by Cruiel, and the author finds it one of the finest in recent works.

The aim of this paper is to propose, and if possible to reveal, the intuitive concept one can imagine about of Lagrangian. By doing so, the author's aim is to first and foremost take away the confusion that students who start to learn analytical mechanics for their first time not to get stuck with Lagrangian while the class keeps its pace to the next topic. This paper is not a result of a research whose goal is to reveal the meaning of Lagrangian. This work is a byproduct of a research conducted to reveal the hidden principles behind animals and human. In other words, this is a byproduct of a research to find out what is and what makes the difference of human movements and robot's movement, a natural one against something artificial to some sense. This paper mathematically evokes the geometrical structure differences between Euler-Lagrange equation obtained from Lagrangian and Canonical equation obtained from Hamiltonian. It claims the naturalness larger in representing a system by Lagrangian formalism rather than in the Hamiltonian formalism, by showing evidence that in some cases the Hamiltonian formalism does not carry an equal geometrical structure of a system derived by Lagrangian formalism which is the same of what is derived by D'Alembert's principle and most of all Newtonian mechanics. The paper comments on the famous classical textbooks on analytical mechanics [2]-[5], how it is to satisfactory or not. It also covers numbers of literatures on Lagrangian, trying to make apparent the significance of it, and a couple works that try the contrary. For the sake of paper length, the author leaves the background to [7]. The author has also tried to look for literatures focused on exactly the meaning of Lagrangian. Unfortunately and to some disappointment, whether due to a premature capacity of the author or the difficulty of covering 240 years of work, no work of such kind could be found. Hence, the author cannot give any additional references, resulting the reference list to being short.

But, there is one study [1] by Morita that briefly proposed a meaning of Lagrangian, short but tackling the issue face-to-face which is not seen in any former literatures. What you can only find that talks about Lagrangian is whether explaining the significance while no one knows the meaning, or emphasize the mysteriousness of it. Morita's work [1] emphasizes on a proposition of a principle criteria behind what is called a Bernstein Problem, which is: how do human solve an ill-posed problem of a "reaching task?" Or in other words, out of the infinite trajectories of the hand path to reach for a coffee cup on a table, how do we choose one trajectory out of it and under what criteria is it chosen? The literature shows the hand path, joint angle histories, joint angular speed history, joint angular acceleration history, and joint torques histories generated by the proposed method, significantly matching with the measured data. While Morita's main focus is to derive a trajectory generation method that is natural in terms of a mechanics point of view, the meaning of Lagrangian is written as a byproduct. In this paper the author will take the byproduct as the main issue and discuss about what could be the meaning of Lagrangian. So the paper discusses the same issue in [1] with vast modification on directly deriving the meaning that yields more clear explanation, where the author finds it worthy to review in depth an already published paper. The process is nothing complicated, rather juvenile the author worries. It is a careful step-bystep review of the elicitation process from equation of motion to the energy equation. Together with [7], this paper will support the statement by Cruiel's work [7] from a totally different perspective that Lagrangian is the most significant and fundamental function in Analytical Mechanics, not Hamiltonian.

In Section 2, the paper begins by deriving the energy equation from the simple equation of motion. This section will claim the proposition of the meaning of the Lagrangian. This section will ban out all the first comers to analytical mechanics from tipping on Lagrangian, using their time wondering what to image till it's too late to study for exams like we and elders has experienced in their days. Section 3 explains that from a certain standpoint, Lagrangian can be perceived as the total kinetic energy, of course in a different sense to the conventional theory that all of us have learned in our elementary years. The author insists here that this section is not to deny any of the conventional theories. It is to show that there is a different way of grasping the energies within a system by the term total kinetic energy. Section 4 explains that the Hamilton's principle is what achieves the theoretical minimum of external energy consumption, widely used today in many areas of industries. Section 3 
will emphasize on the kinetic energy and the potential energy and work from a physical meaning perspective. The difference of Lagrangian and Hamiltonian is in whether you subtract the potential energy or add it. This difference refers to the viewpoint in which one is looking at each entities' energy. Section 5 will point out a foul-up that readers may trip into after perceiving the former sections. Last section will be a summary. The author asks the readers to understand that the attempt here is to give a meaning to a function that, at least till today, was not given any. By giving a meaning to a meaning-absent function will not deny, disrupt, or influence the further mathematical achieved physics, though there maybe cases where one can and should reconsider the physical meaning of the achieved mathematical expressions.

\section{Values You Get When Deriving Energy Equation by Integrating Equation of Motion}

Everyone who learns physics first encounters Newton's Second Law of motion, the equation of motion,

$$
m \ddot{\boldsymbol{q}}=\boldsymbol{F} .
$$

Here $m$ is the mass of the subject under consideration (for convenience a point mass), $\boldsymbol{q}$ representing the position vector of the point mass under consideration, and we shall put it as the generalized coordinate in the configuration space for latter convenience. ( $)$ denotes time derivation. Lastly, $\boldsymbol{F}$ stands for any kind of force acting on the subject. We will stick with this point mass system to avoid unnecessary complication. By doing this will not affect the generality of the discussion about Lagrangian and Hamiltonian or in other words value in order of energy $(\mathrm{N} \cdot \mathrm{m})$. To obtain the information of energy, we learn to first multiply $\dot{\boldsymbol{q}}$ to both sides of Equation (1), and then integrate both sides by time parameter $t$ as,

$$
\begin{gathered}
m \ddot{\boldsymbol{q}} \cdot \dot{\boldsymbol{q}}=\boldsymbol{F} \cdot \dot{\boldsymbol{q}} \\
\Rightarrow \int_{t_{0}}^{t_{1}} m \ddot{\boldsymbol{q}} \cdot \dot{\boldsymbol{q}} \mathrm{d} t=\int_{t_{0}}^{t_{1}} \boldsymbol{F} \cdot \frac{\mathrm{d} \boldsymbol{q}}{\mathrm{d} t} \mathrm{~d} t \\
\Leftrightarrow \int_{t_{0}}^{t_{1}} \mathrm{~d}\left(\frac{m}{2}|\dot{\boldsymbol{q}}(t)|^{2}\right)=\int_{\boldsymbol{q}_{t_{0}}}^{\boldsymbol{q}_{t_{1}}} \boldsymbol{F} \cdot \mathrm{d} \boldsymbol{q} .
\end{gathered}
$$

The right hand side of Equation (4) is the work done by the force $\boldsymbol{F}$ moving the point mass $m$. Now, when there is a $U(\boldsymbol{q})$ where,

$$
\boldsymbol{F}=-\frac{\partial U^{\mathrm{T}}}{\partial \boldsymbol{q}},
$$

then, $\boldsymbol{F}$ is called a conservative force while $U(\boldsymbol{q})$ is called its potential. Using $U(\boldsymbol{q})$, the work of $\boldsymbol{F}$ has the relationship,

$$
\int_{\boldsymbol{q}_{t_{0}}}^{\boldsymbol{q}_{t_{1}}} \boldsymbol{F} \cdot \mathrm{d} \boldsymbol{q}=-U\left(\boldsymbol{q}_{t_{1}}\right)-\left\{-U\left(\boldsymbol{q}_{t_{0}}\right)\right\} .
$$

From Equation (4),

$$
\begin{gathered}
\int_{t_{0}}^{t_{1}} \mathrm{~d}\left(\frac{m}{2}|\dot{\boldsymbol{q}}(t)|^{2}\right)=\int_{\boldsymbol{q}_{t_{0}}}^{\boldsymbol{q}_{t_{1}}} \boldsymbol{F} \cdot \mathrm{d} \boldsymbol{q} \\
\Leftrightarrow \frac{m}{2}\left|\dot{\boldsymbol{q}}_{t_{1}}\right|^{2}-\frac{m}{2}\left|\dot{\boldsymbol{q}}_{t_{0}}\right|^{2}=\int_{\boldsymbol{q}_{t_{0}}}^{\boldsymbol{t}_{t_{1}}} \boldsymbol{F} \cdot d \boldsymbol{q} .
\end{gathered}
$$

Putting $m\left|\dot{\boldsymbol{q}}_{t_{0}}\right|^{2} / 2=0$ affects nothing to the generality of the discussion, and also implementing some adequate constant Const., we can further go,

$$
\frac{m}{2}|\dot{\boldsymbol{q}}(t)|^{2}=\int_{\boldsymbol{q}_{t_{0}}}^{q_{t_{1}}} \boldsymbol{F} \cdot \mathrm{d} \boldsymbol{q}+\text { Const. }
$$

If $\boldsymbol{F}$ is conservative, then Equation (9) can further go,

$$
\frac{m}{2}|\dot{\boldsymbol{q}}(t)|^{2}=-U(\boldsymbol{q}(t))+\text { Const } .
$$


Equation (10) does not lose generality by putting Const. =0 so Equation (10) can further be transformed to,

$$
\frac{m}{2}|\dot{\boldsymbol{q}}(t)|^{2}=-U(\boldsymbol{q}(t)) \text {. }
$$

If one demands to make the issue up to a more general case, they can insert a nonconservative force $f$ on the right hand side of Equation (1),

$$
m \ddot{\boldsymbol{q}}=\boldsymbol{F}+\boldsymbol{f} .
$$

Now you will have another player within the system whose work is $\int_{\boldsymbol{q}_{t_{0}}}^{q_{t_{1}}} \boldsymbol{f} \cdot \mathrm{d} \boldsymbol{q}$ in terms of energy. So,

$$
\frac{m}{2}|\dot{\boldsymbol{q}}|^{2}=-U(\boldsymbol{q})+\int_{\boldsymbol{q}_{t_{0}}}^{\boldsymbol{q}_{\mathrm{t}_{1}}} \boldsymbol{f} \cdot \mathrm{d} \boldsymbol{q},
$$

holds. Equation (13) means $\boldsymbol{F}$ and $\boldsymbol{f}$ have labored $-U(\boldsymbol{q})+\int_{\boldsymbol{q}_{t_{0}}}^{q_{\mathrm{t}_{1}}} \boldsymbol{f} \cdot \mathrm{d} \boldsymbol{q}$ making the mass $m$ to be $m|\dot{\boldsymbol{q}}(t)|^{2} / 2$ much vital. Equation (13) can be perceived as a transfer of energy from one kind to another.

Equation (13) gives you mainly three informations;

(a) $m|\dot{\boldsymbol{q}}(t)|^{2} / 2$ is the kinetic energy of the mass under the subject, showing how vital the mass is moving. Here, we only know the magnitude of how active the mass is moving, the term does not give us any more information in detail, for example in which direction is it moving or accelerating.

(b) One can interpret Equation (13) that it took $-U(\boldsymbol{q}(t))+\int_{\boldsymbol{q}_{t_{0}}}^{\boldsymbol{q}_{t_{1}}} \boldsymbol{f} \cdot \mathrm{d} \boldsymbol{q}$ of work by force $\boldsymbol{F}$ and $\boldsymbol{f}$ to make the mass as vital as $m|\dot{\boldsymbol{q}}|^{2} / 2$. In other words, Equation (13) represents a transfer of energy from one kind to another. In this case both $\boldsymbol{F}$ and $\boldsymbol{f}$ 's work was transferred to $m$ 's kinetic energy.

(c) In terms of Lagrangian and Hamiltonian, nonconservative forces are in consideration. Hence, if we focus only about cases where there is no other kind of force except conservative force, $m|\dot{\boldsymbol{q}}(t)|^{2} / 2$ and $-U(\boldsymbol{q}(t))$ are equal in terms of value. Not $U(\boldsymbol{q}(t))$.

Plus, Equation (5) indicates;

(d) Potential energy is just a special name given to a certain type of energy. Even if something such as $\boldsymbol{F}=-\partial U / \partial \boldsymbol{q}$ holds, $-U(\boldsymbol{q})$ is always in a class of work [4]. It may be called a potential energy but it will always be within the category of work.

Given that we are considering only conservative force within the system, (a) through (d) indicates at time $t$ the mass $m$ became as vital as to have $m|\dot{\boldsymbol{q}}|^{2} / 2$ much of energy, and force $\boldsymbol{F}$ has worked $-U(\boldsymbol{q})$ much of energy. The usual explanation will be that the work done by the force $\boldsymbol{F}$ has transferred to the kinetic energy of $m$, thus the only amount of energy with in the system is either $m|\dot{\boldsymbol{q}}(t)|^{2} / 2$ or $-U(\boldsymbol{q}(t))$.

Meanwhile in Lagrangian style, all players within the system is equally and independently recognized (Figure 2). The concern is on every entity listed as in Table 1. So, simply taking the net sum of every type of energy

\begin{tabular}{|c|c|c|c|}
\hline Subject & (Entity) & Value & Type \\
\hline$m:$ & Mass & $m|\dot{\boldsymbol{q}}|^{2} / 2$ & Kinetic energy of mass (work against void space) \\
\hline$m g$ : & Gravity & $\int m \boldsymbol{g} \cdot \mathrm{d} \boldsymbol{q} \quad(=-m|\boldsymbol{g}| h \quad h:$ hight $)$ & Work of gravity (gravitational potential) \\
\hline$F:$ & Conservative force & $\int \boldsymbol{F} \cdot \mathrm{d} \boldsymbol{q}(=-U(\boldsymbol{q}))$ & Work of conservative force \\
\hline$f:$ & Nonconservative force & $\int f \cdot \mathrm{d} \boldsymbol{q}$ & Work of nonconservative force \\
\hline
\end{tabular}
with in the system,

Table 1. Energies and its types within a given system in Figure 1 and Figure 2. 


$$
\frac{m|\dot{\boldsymbol{q}}(t)|^{2}}{2}+(-m|\boldsymbol{g}| h)-U(\boldsymbol{q}(t))+\int_{\boldsymbol{q}_{t_{0}}}^{\boldsymbol{q}_{t_{1}}} \boldsymbol{f} \cdot \mathrm{d} \boldsymbol{q},
$$

is what is to be obtained. This view neglects whether there is a consumption occurring or an energy is transferred to a different type of energy and etc. It just wants to see what is there. Speaking anthropomorphic, it's an information of all the actors within the whole play. In this case the whole play is the physical system where something is happening, and the information of all the actors is given simply as an addition of them in terms of energy and work. Even with the kinetic energy, it could be seen as a kind of work if you like. If you feel uncomfortable, then what if the mass $m$ is an exterior force pushing another mass, say $m^{\prime}$ ? One can always know the force of a mass $m$ from $f=m \ddot{q}$. For $m^{\prime}, m$ 's kinetic energy is exactly the amount of work it would get. By allowing this way of looking at a mass, it could be said that a mass $m$ is applying "work" $m|\dot{\boldsymbol{q}}| / 2$ against a void space. Just because there is nothing to apply ones "work" does not implicate that such case is cannot occur. An astronaut in a space station doing a sit up exercise without a support will definitely make him tired. But then who receives extra energy? No one. Just because there's no one to receive a work does not mean the subject is not working. That is why the mass in Table 1 has its type as a "(work against void space)." Under this viewpoint, if one is considering a system with a mass $m$ and just a conservative force $\boldsymbol{F} \quad\left(=-(\partial U / \partial \boldsymbol{q})^{T}\right)$, then the total sum will be,

$$
\frac{m|\dot{\boldsymbol{q}}(t)|^{2}}{2}-U(\boldsymbol{q}(t))
$$

which is exactly the function structure of a Lagrangian. Being anthropomorphic once again, Lagrangian is like the work of the whole system as if it is like a laborer. Thus the action integral is like the whole effort of the laborer of a one day's work (a physical reaction happening within a certain time interval) and the Hamilton's principle indicates that mechanics imposes the entities within the system to act and move so that the total effort of the laborer would be the minimum (stationary, being more precise).

This is totally different from a Hamiltonian way $\left(m|\dot{\boldsymbol{q}}(t)|^{2} / 2+U(\boldsymbol{q}(t))\right)$ of looking at a system from an energy view. Trying best to picture the situation with Figure 1, Hamiltonian way of perceiving a system is not the net sum of all the energy there is within the system, it sees how much energy is there within the system at a given moment, for example,

$$
\frac{m|\dot{\boldsymbol{q}}(t)|^{2}}{2}+m|\boldsymbol{g}| h+U(\boldsymbol{q}(t))-\int_{\boldsymbol{q}_{t_{0}}}^{\boldsymbol{q}_{t_{1}}} \boldsymbol{f} \cdot \mathrm{d} \boldsymbol{q} .
$$

It just wants to see what is left within the system in terms of energy. As done in Lagrangian style the total energy existing within a system is, only considering a system with a mass $m$ and a conservative force $\boldsymbol{F}$ $\left(=-(\partial U / \partial \boldsymbol{q})^{T}\right)$,

$$
\frac{m|\dot{\boldsymbol{q}}(t)|^{2}}{2}+U(\boldsymbol{q}(t))
$$

which is exactly the function structure of a Hamiltonian.

The reason why Lagrangian is structured with a minus sign on the potential energy term is because of the way one is observing the system (Figure 1, Figure 2). This corresponds to how Lagrangian is derived from D'Alembert's principle, which handles energy of any entity within the system without any distinction. Hence, it makes no inconsistencies with the D'Alembert's principle. A more useful reason why some physical function is structured that way is discussed in Section 4.

\section{Which Is Total Kinetic Energy? Hamiltonian or Lagrangian}

Now, let us recall the Hamiltonian and total kinetic energy $m|\dot{\boldsymbol{q}}(t)|^{2} / 2+U(\boldsymbol{q}(t))$. As discussed in Section 2, Lagrangian is a collection of all the entities' energy within a system expressed as a simple sum which is literally total energy within a system. Then which one is more legitimate to be called "total kinetic energy," Hamiltonian or Lagrangian? The answer is, they both do. 

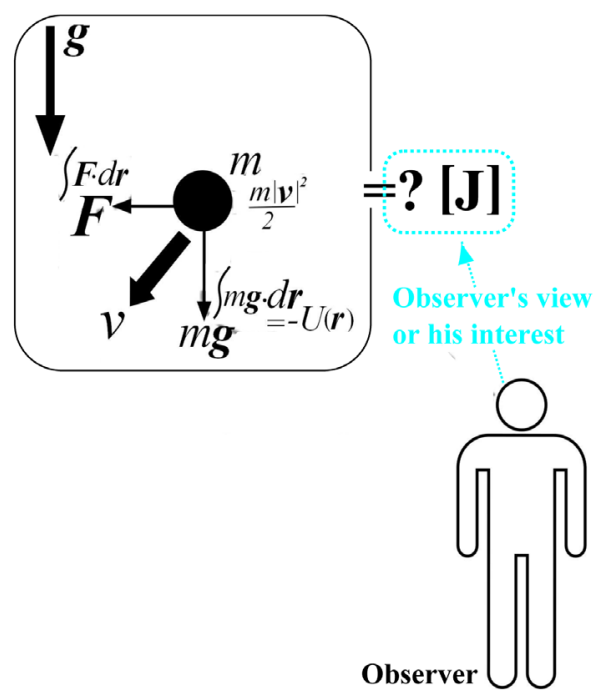

Figure 1. Illustration of a Hamiltonian viewpoint image.

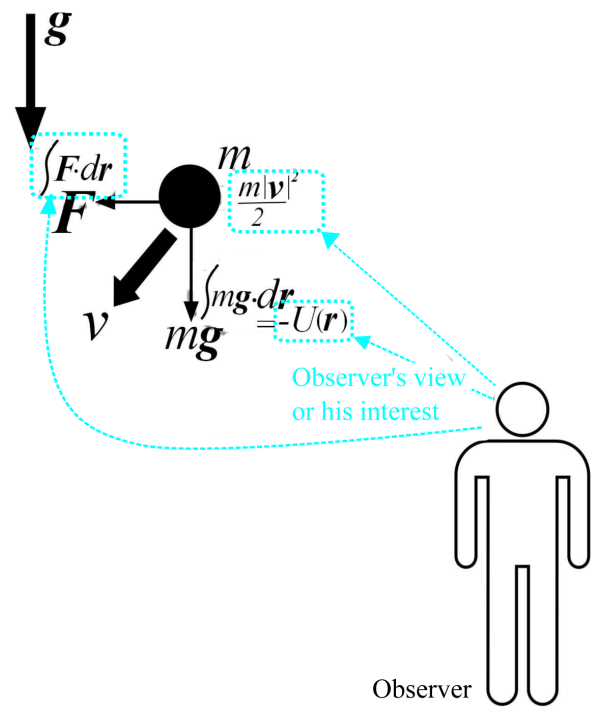

Figure 2. Illustration of a Lagrangian viewpoint image.

Somehow, Lagrangian bought the price of being a enigma while Hamiltonian was fitted in so smoothly due to the occasion that the first integral of time symmetry of a system was equal to the total kinetic energy. One may think that it would have turned around if potential energy was defined without a minus sign, $\boldsymbol{F}=\partial U / \partial \boldsymbol{q}$. But that is not the case because, if it were defined as such the total kinetic energy would have been $m|\dot{\boldsymbol{q}}|^{2} / 2-U(\boldsymbol{q})$, and Lagrangian would have been found in a style as $m|\dot{\boldsymbol{q}}|^{2} / 2+U(\boldsymbol{q})$. The Legandre transform of the Lagrangian would define the Hamiltonian as $m|\dot{\boldsymbol{q}}|^{2} / 2-U(\boldsymbol{q})$, thus making the same situation with a different sign before the potential energy term. So, without discriminating the view point of the observer described in Section 2, there is no way to give a imaginable meaning to the Lagrangian. So redefining potential energy without a minus sign is not a fundamental remedy of the last 240 years of students suffering. This is how it has been and will be unless the reader copes with the proposal of this paper.

As a summary, Hamiltonian and Lagrangian are both legitimate to be called a total kinetic energy, but they differ in the attitude of the observer looking at the system and its entities. Hamiltonian is a function that gives you the income and outgo of energy within the system. The value does not care of the energy type transfers 
occurring in the system. The value does not care about the happenings inside the system. Lagrangian does not focus on the income-outgo of the systems' energy, it handles kinetic energy and potential energy in an equal manner. Hence, the action integral can be understood as a time integration of every and any kind of energy, whether a kinetic energy or a potential energy or work of some force acting within the system, during the whole motion. So turns out that Hamilton's principle is in words finding a path $\boldsymbol{q}(t)$ that gives an extremum of the sum of all the energy one can find within a system integrated through the whole duration. It can be interpreted as a principle requiring the combination that yields the least expenditure of the sum of all the energy that can be found within the system during a motion.

\section{The Action Integral Is the Very Definition of the Least Energy Consumption}

As a byproduct of the discussion, we obtain a theoretical (possibly) minimum energy consumption condition for a certain task. Let us consider work $W$ of a nonconservative force $f$, with its potential $U$, and a point mass that has a kinetic energy $K$ at the given moment. Then, the extended Hamilton's principle [5] is,

$$
\delta \int_{t_{0}}^{t_{1}} K-U+W \mathrm{~d} t=0 .
$$

Equation (18) can further be transformed as,

$$
\delta \int_{t_{0}}^{t_{1}} K-U \mathrm{~d} t=-\delta \int_{t_{0}}^{t_{1}} W \mathrm{~d} t
$$

If the both sides of Equation (19) is equal to 0, then it is the case of a free motion, a motion with only conservative forces acting on the point mass because $\delta \int_{t_{0}}^{t_{1}} K-U \mathrm{~d} t=0$ is exactly a form of Hamilton's principle. In this case, both,

$$
\left\{\begin{array}{l}
\delta \int_{t_{0}}^{t_{1}} K-U \mathrm{~d} t=0 \\
\delta \int_{t_{0}}^{t_{1}} W \mathrm{~d} t=0
\end{array}\right.
$$

holds, meaning that $\int_{t_{0}}^{t_{1}} W \mathrm{~d} t$ and $\int_{t_{0}}^{t_{1}} K-U \mathrm{~d} t$ is at a minimum (or more precisely stationary) situation. Equation (20) both holds at the same time, therefore solving $\delta \int_{t_{0}}^{t_{1}} W \mathrm{~d} t=0$ and $\delta \int_{t_{0}}^{t_{1}} K-U \mathrm{~d} t=0$ is about acquiring the same solution. $\delta \int_{t_{0}}^{t_{1}} W \mathrm{~d} t=0$ means that the total effort by force $F$ is at a minimum (or more precise at an extremum). Plus that $\delta \int_{t_{0}}^{t_{1}} K-U \mathrm{~d} t=0$ is exactly the Hamilton's principle, Hamilton's principle is a principle to obtain minimum energy consumption. A simple variational calculation to $\delta \int_{t_{0}}^{t_{1}} W \mathrm{~d} t=\delta \int_{t_{0}}^{t_{1}} \boldsymbol{f} \cdot \mathrm{d} \boldsymbol{q}=0$ will yield nonconservative force $\boldsymbol{f} \equiv \mathbf{0}$ denoting that the motion is a free motion which makes perfect sense. One can also appreciate this situation as the least energy consumption because $\boldsymbol{f} \equiv \mathbf{0}$ holds during the whole duration, consuming no external energy at all. Equation (20) are both necessity condition for a minimum (nonconservative force's) energy consumption. But $\boldsymbol{f} \equiv \mathbf{0}$ is all we can get, then only free motion is eligible so we can do nothing to the system. But if there is a way to find a series of $\boldsymbol{q}(t)$ where $\boldsymbol{f}(t) \neq \mathbf{0}$, then such $\boldsymbol{q}(t)$ and $f(t)$ could possibly yield an minimum energy consuming motion, which is highly recommended in industries. One may wonder how occasion where (20) and $\boldsymbol{f}(t) \neq \mathbf{0}$ can be achieved. Such $\boldsymbol{q}(t)$ and $\boldsymbol{f}(t)$ can be found in [1]. So, it is quite worthy on discussing about the physical meaning of Lagrangians.

\section{Misconception and Misunderstanding That Occur from the Former Discussions and Previous Eras' Education}

\subsection{Lagrangian Cannot Have a Meaning Because It Is Not Unique?}

Like the gauge transformation [4] tells us, Lagrangian is not necessary unique. For some given function $g$ where it is a function of the generalized coordinates $\boldsymbol{q}$ and time $t$ and it is differentiable by $t$, a new Lagrangian $L^{\prime}$ that yields the same Euler-Lagrange equation from predefined Lagrangian $L$ can always newly be made by, 


$$
L^{\prime}=L+\frac{\mathrm{d} g(\boldsymbol{q}, t)}{\mathrm{d} t} .
$$

The author asks the readers to think about the $g$ induced with in Equation (21). Where in terms of information do you get to obtain the function $g$ ? Yes, such $g$ does no harm in deriving the demanded Euler-Lagrange equation. But that is in mathematical terms, not by any means supported by physics. The author's claim here is that most of $L^{\prime}$ derived by $g$ is quite artificial in the sense that $g$ is not necessarily a function derived from the systems information. For instance, like gauge transformation of an electromagnetic field system seen in many textbook exercises is not artificial in the author's sense, because the $g$ chosen for this example shows that $g$ carries with it information on the physical system under consideration. But if you have a function $g$ that carries with it no information of the system at all, it is only mathematically legitimate for deriving the same EulerLagrange equation. Why is it wrong to recognize the two as of a different class? Or why should we treat them with the same status in terms of physics?

The author received a notion that one cannot give a unique meaning to something that multiply exists. This was shocking because this is quite out of the argument appropriate in a level of professionals. The claim was saying as if that defining an airplane as "a man-build vehicle that flies in the air with a fixed wing" is a wrong doing, not because the definition is not accurate enough but because there is a Cessna, Beechcraft, Boeing 787, Concords, Airbus 380, etc. multiple aircrafts in the world. This is totally out of logic. He may have claimed it in a different sense that because each of the arbitrary Lagrangians may have different meaning, therefore giving a unique meaning to the whole class of the arbitrary Lagrangians is not possible. This claim is totally logical, and the author has no objection. But one must recognize that the claim does not deny some particular Lagrangians having a unique meaning just only applicable to themselves. If one denies this, then what would be the evidence in categorizing ourselves human? It is wrong in classifying Mary and Jack both human beings?

\subsection{Potential Energy Is Not Work?}

The author have had a dispute that calling potential energy as a type of work is by definition wrong. The two has a complete different property. The author agrees that the two has a different property, but author disagrees the dispute that potential energy is not a type of work.

This is pretty shocking because we have to go all the way back to the most elementary state of learning mechanics. To all those that thinks potential energy is not work, the author begs them to open their introductory textbook on mechanics, such as [4], to the chapter on energy and work. There one should find first that a work $W$ of a given force $\boldsymbol{F}$ is defined by,

$$
W=\int_{\boldsymbol{q}_{t_{0}}}^{\boldsymbol{q}_{\mathrm{t}_{1}}} \boldsymbol{F} \cdot \mathrm{d} \boldsymbol{q} .
$$

The above applies for any and all kinds of force, hence of course conservative forces like gravity and electro-magnetic forces are included. The above as a premise, potential energy is defined when force $\boldsymbol{F}$ has a property when there is a scalar function $U(\boldsymbol{q})$ where,

$$
\boldsymbol{F}=-\frac{\partial U}{\partial \boldsymbol{q}} .
$$

When the above holds then,

$$
W=\int_{\boldsymbol{q}_{0}}^{\boldsymbol{q}_{1}} \boldsymbol{F} \cdot \mathrm{d} \boldsymbol{q}=\int_{\boldsymbol{q}_{0}}^{\boldsymbol{q}_{1}}\left(-\frac{\partial U(\boldsymbol{q})}{\partial \boldsymbol{q}}\right) \mathrm{d} \boldsymbol{q}=-\int_{\boldsymbol{q}_{0}}^{\boldsymbol{q}_{1}} \mathrm{~d} U=-U(\boldsymbol{q}),
$$

holds. When there exists such $U(\boldsymbol{q})$, it is called a potential energy. As you can see from the derivation above, the definition of $U(\boldsymbol{q})$ as a potential energy does not deny the fact that it can be perceived as a form of work. Or rather, the definition of a potential energy is given as a special case of work with an opposite sign. The author finds no serious logical disruption.

\subsection{Hamilton's Principle Could Change into a Silly Simple Expression}

Let us introduce $K\left(:=m|\dot{\boldsymbol{q}}(t)|^{2} / 2\right)$ for kinetic energy in general. Then, as we seen in former sections 
Lagrangian $L$ is,

$$
L=K-U \text {. }
$$

From $-U(\boldsymbol{q})=\int \boldsymbol{F} \cdot \mathrm{d} \boldsymbol{q}$ with Equation (4), Equation (11) indicating $m|\dot{\boldsymbol{q}}|^{2} / 2=K=-U$, the above could be further manipulated as,

$$
\begin{aligned}
& L=K-U \\
& =2 K \\
& =2 \int \boldsymbol{F} \cdot \mathrm{d} \boldsymbol{q} \\
& =-2 U .
\end{aligned}
$$

Remember these manipulations hold not just for a system with a point mass but any kind of physical system, as long as they are in the size where Newtonian mechanics is appropriate. As Equation (23) or Equation (24) tells, any system at any given time that Lagrangian is always twice of the kinetic energy of the subject under consideration or twice that of the work done to the subject under consideration.

There maybe readers who wonder such as,

Why not minimize $K$ or $-U$ instead? Equation (23) and Equation (24) denotes that $L=2 K=-2 U$ and moreover, $K$ is a convex function. It's very convenient.

If you come up with this question, you must understand the meaning of an equal sign "=” in mathematics, more precisely arithmetics. $A=B$ means that $A$ and $B$ are equal, yes. But that is in the sense of values and numbers, and solely that. This is more understandable if it was written like $A(x)=B(x, y)$. This still tells us that both $A(x)$ and $B(x, y)$ are equal, but only as numeric values, not its function structure. That is why minimizing solely $K$ or $-U$ will not be the same as minimizing $L(=K-U)$, the Lagrangian.

\section{Conclusions}

This paper pointed out the followings;

a) One can perceive Lagrangian with a conceptual meaning.

b) Lagrangian is the effort of the potential energy working on a physical subject and that physical subject's kinetic energy showing its own intensity of moving, or in other words, the physical subject working against a void space, at a given moment.

c) Hamilton's principle is the very definition of the least energy consumption.

Following these were some misunderstandings that may occur in accepting that the content of the paper is noted and untangled. An important thing not noted apparently in this paper is that a Lagrangian having a perceivable meaning does not deny any of the mechanics and physics that has been constructed over the 240 years after the Lagrangian's introduction.

With this paper, the author hopes many coming first learners of analytical mechanics not to get stuck on Lagrangian, leave it as just a very useful tool, but imagine the concept in vision like you could with many other physical values like kinetic energy and inertia and so on. What may be derived from understanding the meaning of a Lagrangian can be found in [1].

\section{Acknowledgements}

We thank the Editor and the referee for their comments. The research of S. Morita does not have a particular funding, grants, and supports, though there is a great gratitude to the university. Finally, an acknowledgment that is out of words goes to S. Morita’s doctrate supervisor Prof. T. Ohtsuka currently at Kyoto University, Japan.

\section{References}

[1] Morita, S. (2012) Trajectory Generation between Two Arbitrary States Based on Hamilton’s Principle-A Variable Substitution Method. International Journal of Humanoid Robotics, 9, 3. http://www.worldscientific.com/doi/pdf/10.1142/So219843612500235 http://dx.doi.org/10.1142/S0219843612500235 
[2] Lifshitz, E.M. and Landau, L.D. (1981) Mechanics. 3rd Edition, Vol. 1, Butterworth-Heinmann, Oxford.

[3] Arnold, V.I. (1997) Mathematical Methods of Classical Mechanics. 2nd Edition, Springer, New York.

[4] Goldstein, H., Poole, C.P. and Safko, J.L. (2013) Classical Mechanics. 3rd Edition, Pearson Education Limited, Essex.

[5] Lanczos, C. (1986) The Variational Principles of Mechanics. 4th Edition, Dover, New York.

[6] Suto, Y. (2008) Analytical Mechanics Quantum Theory. University of Tokyo Press, Tokyo. (In Japanese)

[7] Cruiel, E. (2014) Classical Mechanics Is Lagrangian; It Is Not Hamiltonian. British Journal for the Philosophy of Science, 65, 269-321. http://www.bjps.oxfordjournals.org/content/early/2013/05/11/bjps.axs034.abstract http://dx.doi.org/10.1093/bjps/axs034 